\title{
Challenges in Current Management of Valvular Heart Disease. Thinking Outside the Box
}

\author{
Locorotondo G*, Diana G*, Crea F** and Massetti $\mathbf{M}^{* *}$ \\ Department of Cardiovascular Sciences, Fondazione Policlinico Universitario A. Gemelli IRCCS, Italy
}

*Corresponding author: Locorotondo G, Department of Cardiovascular Sciences, Fondazione Policlinico Universitario A. Gemelli IRCCS, Largo A. Gemelli 8, 00168 Rome, Italy.

To Cite This Article: Locorotondo G*, Diana G, Crea F, Massetti M. Challenges in Current Management of Valvular Heart Disease. Thinking Outside the Box. 2020 - 9(4). AJBSR.MS.ID.001410. DOI: 10.34297/AJBSR.2020.09.001410.

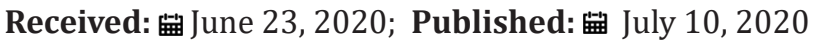

\section{Opinion}

Management of valvular heart disease (VHD) is a continuously evolving process: since a prosthetic mechanical valve was firstly implanted in humans, technical advancements in diagnosis and treatment of VHD have taught us that we should always keep the well-proven and be open-minded to something new. According to current guidelines, management of VHD is mainly based on an intricate puzzle, relying on fine diagnosis, accurate prognostic stratification and tailored treatment. Despite all efforts, some gaps in knowledge still exist concerning appropriate patient selection and correct timing of treatment, which sometimes leads us to consider the need of "thinking outside the box" in current clinical practice.

The tale of functional mitral regurgitation (FMR) is a clear example of the need of "thinking outside the severity". While treatment options for primary mitral regurgitation (MR) are straightforward and lead almost invariably to good outcomes, chronic secondary MR is associated with a high risk of poor prognosis [1], regardless of treatment [2]. A percutaneous edgeto-edge procedure is indicated as a treatment option when revascularization surgery is not needed and the patient is judged to be too high risk. However, the two randomized controlled trials, MITRA-FR [3] and COAPT [4], performed to evaluate the clinical efficacy and safety of percutaneous mitral-valve repair in addition to medical treatment in patients with heart failure (HF) and FMR, have given opposing results, with the former showing that the rate of the composite primary outcome of death or unplanned hospitalization for heart failure at 12 months did not differ between intervention and control groups, and the latter showing that the device-based treatment resulted in a significantly lower rate of hospitalization for HF, lower mortality, and better quality of life and functional capacity within 24 months of follow-up than medical therapy alone. Examining the baseline characteristics of the two patient populations gives us an important lesson on how selection of patient and correct timing of treatment may impact on outcome. Indeed, in the MITRA-FR trial, patients had striking left ventricle (LV) dilatation with MR proportional to the degree of LV dilatation, whereas, in the COAPT trial, investigators enrolled patients in whom the degree of MR was disproportionately greater compared with the degree of LV chamber enlargement. Such a characterization of FMR, which has been previously defined as "proportionate" and "disproportionate" MR [5], actually should represent a new framework of optimal timing of treatment, i.e. when there is a severe FMR in a not-severely dilated LV. Expectations are for the results of RESHAPE-HF2 (NCT02444338), an ongoing European clinical trial, designed to better clarify the effectiveness of percutaneous edgeto-edge mitral repair.

Contrary to FMR, treatment of aortic stenosis (AS), to date, represents an example of the need of "thinking outside the symptoms", as management of asymptomatic patients remains controversial. According to guidelines early elective surgery is indicated only in asymptomatic patients with depressed LV ejection fraction (LVEF) not due to other causes and in patients who develop symptoms during exercise testing. However, many patients with severe AS have impaired LV systolic function, expressed by global longitudinal strain (GLS), despite normal LVEF [6]: in such patients, survival probability is significantly reduced, compared to patients with normal GLS. Particularly, impairment of GLS carries a mortality risk similar to that of severe AS with impaired LVEF, and patients with low GLS despite normal LVEF have a 20-fold higher mortality risk than patients with normal GLS and normal LVEF [7]. The key feature that leads to heart failure in patients with aortic stenosis is myocardial fibrosis, a consequence of the progressive narrowing of the valve and the progressive increase of the afterload. In patients with aortic stenosis, cardiac magnetic resonance is able to 
accurately distinguish a late replacement fibrosis stage, irreversible and therefore associated with unfavorable outcomes despite aortic valve replacement (AVR), and an earlier stage of diffuse reactive fibrosis, potentially reversible after AVR [8]. Although unable to distinguish between reactive and replacement fibrosis, GLS strictly correlate with it, as its impairment is associated with an increase of myocardial fibrosis. Notably, GLS shares a parallel tale with myocardial fibrosis, as in most, but not all, patients it improves after AVR. Novel strain parameters have been proposed with the aim to predict which patients will most likely recover systolic function after AVR [9]. These issues should encourage the next steps aimed at trying to understand if an earlier treatment strategy, based on the non-invasive study of myocardial fibrosis, could have an impact on the outcomes in patients with aortic stenosis, and the ongoing EvoLVeD trial (NCT03094143) has been designed with such a purpose. No less ambitious would also be the aim of establishing the possibility of strain echocardiographic parameters to discriminate reactive from replacement fibrosis.

Finally, sometimes it is needed to "thinking outside the valve": it is the case of mitral valve prolapse (MVP), that may be associated with poor prognosis due to its high risk of malignant arrhythmias regardless of the degree of mitral regurgitation. MVP is an underestimated significant cause of arrhythmic sudden cardiac death and life-threatening arrhythmias. The prolapsing leaflet triggers myocardial stretch leading to fibrosis of the papillary muscles and infero-basal left ventricular wall which represents the structural hallmark of ventricular arrhythmias. An important role also seems to be played by mitral annulus disjunction (MAD), a constant feature of arrhythmic MVP with LV fibrosis $[10,11]$. It is associated with a high occurrence of ventricular arrhythmias, also in non-prolapse mitral valve disease [12]. Myocardial fibrosis and MAD should therefore be carefully evaluated in patients with MVP even in the absence of hemodynamic impairment or severe mitral valve regurgitation. Their acknowledgement should lead to close monitoring of patients and the use of early arrhythmia prevention strategies in order to reduce their risk of SCD.

In conclusion these recent evidence highlight the relevance of "thinking outside" the current indications for the management of VHD in specific clinical scenarios to better define timing, strategy of treatment and risk stratification of patients. Ongoing trials are expected to further expand recommendations beyond those currently taken into account. In the meantime, as clearly suggested by guidelines, a heart team approach seems to be the best way towards a really personalized medicine.

\section{Reference}

1. Trichon BH, Felker GM, Shaw LK, Cabell CH, O Connor CM (2003) Relation of Frequency and Severity of Mitral Regurgitation to Survival Among Patients With Left Ventricular Systolic Dysfunction and Heart Failure. Am J Cardiol 91(5): 538-543.

2. Blasé A Carabello (2008) The Current Therapy for Mitral Regurgitation. Journal of the American College of Cardiology 52(5): 319-326.

3. Obadia JF, Messika Zeitoun D, Leurent G, Iung B, Bonnet G, et al. (2018) Percutaneous Repair or Medical Treatment for Secondary Mitral Regurgitation. NEJM 379(24): 2297-2306.

4. Stone GW, Lindenfeld J, Abraham WT, Kar S, Lim DS, et al. (2018) Transcatheter Mitral-Valve Repair in Patients with Heart Failure. NEJM 379(24): 2307-2318.

5. Grayburn PA, Sannino A, Packer M (2019) Proportionate and Disproportionate Functional Mitral Regurgitation A New Conceptual Framework That Reconciles the Results of the MITRA-FR and COAPT Trials. JACC Cardiovasc Imaging 12(2): 353-362.

6. Kearney LG, Lu K, Ord M, Patel SK, Profitis K, et al. (2012) Global longitudinal strain is a strong independent predictor of all-cause mortality in patients with aortic stenosis. European Heart Journal Cardiovascular Imaging 13(10): 827-833.

7. Ng ACT, Prihadi EA, Antoni ML, Bertini M, Ewe SH, et al. (2017) Left ventricular global longitudinal strain is predictive of all-cause mortality independent of aortic stenosis severity and ejection fraction. European Heart Journal - Cardiovascular Imaging 19(8): 859-867.

8. Bing R, Cavalcante JL, Everett RJ, Clavel MA, Newby DE, et al. (2019) Imaging and Impact of Myocardial Fibrosis in Aortic Stenosis. JACC cardiovascular Imaging 12(2): 283-296.

9. Slimani A, Melchior J, De Meester C, Pierard S, Roy C, et al. (2020) Relative Contribution of Afterload and Interstitial Fibrosis to Myocardial Function in Severe Aortic Stenosis. JACC Cardiovascular imaging 13(2 Pt 2): $589-600$.

10. Basso C, Perazzolo Marra M, Rizzo S, De Lazzari M, Giorgi B, et al. (2015) Arrhythmic Mitral Valve Prolapse and Sudden Cardiac Death. Circulation 132(7): 556-566.

11. Perazzolo Marra M, Basso C, De Lazzari M, Rizzo S, Cipriani A, et al. (2016) Morphofunctional Abnormalities of Mitral Annulus and Arrhythmic Mitral Valve Prolapse. Circ Cardiovasc Imaging 9(8): e005030.

12. Dejgaard LA, Skjølsvik ET, Lie $\varnothing \mathrm{H}$, Ribe M, Stokke MK, et al. (2018) The Mitral Annulus Disjunction Arrhythmic Syndrome. JACC 72(14): 16001609. 